\begin{tabular}{|c|l|}
\hline Title & $\begin{array}{l}\text { Numerical Simulation of Gas Liquid Two-Phase Flow in a Horizontally Placed Hydrophobic Rectangular Channel (Part } \\
\text { 2, Influence of A brupt Contraction) }\end{array}$ \\
\hline Author(s) & Ueda, Y.; Nakajima, T.; Ishii, T.; T sujino, R.; Iguchi, Manabu \\
\hline Citation & $\begin{array}{l}\text { High Temperature Materials and Processes, 31(4-5), 411-413 } \\
\text { https://doi.org/40.1515/hmp-2012-0075 }\end{array}$ \\
\hline Issue Date & 2012-10 \\
\hline Doc URL & http:/hdl.handle.net/2115/53465 \\
\hline Rights & The final publication is available at www.degruyter.com \\
\hline Type & article \\
\hline File Information & HTMP31-45_411-413.pdf \\
\hline
\end{tabular}

Instructions for use 
Y. Ueda*, T. Nakajima, T. Ishii, R. Tsujino and M. Iguchi

\section{Numerical Simulation of Gas-Liquid Two-Phase Flow in a Horizontally Placed Hydrophobic Rectangular Channel (Part 2, Influence of Abrupt Contraction)}

Abstract: This paper computationally visualizes twophase flow patterns through a horizontally placed hydrophilic or hydrophobic rectangular channel with an abrupt contraction. The rectangular duct used in this study has a thickness narrower than the Laplace constant so that the surface tension governs the fluid system rather than the inertia force. In particular, the computed bubble behavior at the abrupt contraction seemed to be a similar nature against the preliminary experimental result.

Keywords: two-phase flow, rectangular channel, wettability, abrupt contraction, CFD

PACS $^{\circledR}$ (2010). 47.15.-x, 47.55.-t, 68.08.Bc

\footnotetext{
*Corresponding author: Y. Ueda: Division of Materials Science and Engineering, Graduate School of Engineering, Hokkaido University, Nishi 8, Kita 13, Kita-Ku, Sapporo, Hokkaido 060-8628, Japan E-mail:y-ueda@eng.hokudai.ac.jp

T. Nakajima: Division of Mechanical Engineering, Graduate School of Engineering, Osaka Prefecture University, 1-1, Gakuen-Cho, Sakai, Osaka 599-8531, Japan

T. Ishii: JFE Steel Corporation, 1-1 Minami-Watarida-Cho, KawasakiKu, Kawasaki, Kanagawa 210-0855, Japan

R. Tsujino: Department of Mechanical Engineering, Setsunan University, 17-8, Ikedanaka-Machi, Neyagawa, Osaka 572-8508, Japan

M. Iguchi: Division of Materials Science and Engineering, Graduate School of Engineering, Hokkaido University, Nishi 8, Kita 13, Kita-Ku, Sapporo, Hokkaido 060-8628, Japan
}

\section{Introduction}

Two-phase flow in a channel is an intriguing issue to control flow systems in complicated piping geometries of nuclear energy plant and steelmaking plant, etc. To design such two-phase flow systems, we need to investigate the influence of abrupt change of a duct area, i.e., abrupt expansion and contraction. The influence of the abrupt expansion has been investigated in the coupled study [1], and so the investigation on the abrupt contraction is required. In particular, a narrow thickness of the rectangular duct affects the nature of the two-phase flow, i.e., if the duct height (not a hydraulic diameter) would be smaller than the Laplace constant $\sqrt{\sigma /\left[g\left(\rho_{L}-\rho_{G}\right)\right]}$, the surface tension governs the fluid system rather than the inertia force. Here, $\rho_{L}$ and $\rho_{G}$ are the densities of liquid and gas, $\sigma$ the surface tension and $g$ the acceleration due to gravity. Similar to the associated study [1], the present setting of the duct thickness is $2 \mathrm{~mm}$ which is smaller than the calculated Laplace constant of about $2.7 \mathrm{~mm}$ in the present situation.

As the preliminary step, this paper computationally visualizes two-phase flow patterns through an abrupt contraction of a hydrophobic or hydrophilic rectangular duct with the narrow thickness.

\section{Computational procedure}

The FLUENT numerical code ver. 6.2.16, a commercially available CFD software package, was employed for all numerical predictions on Intel Core 2 Quad $2.66 \mathrm{GHz}$ processor with 3.25 GB RAM. GAMBIT 2.2.30 was employed for the establishment of the three-dimensional computational grid. The detailed description about the actual setting of the FLUENT code is given in the associated study [1].

The computational grids were made up of structured elements and a total of 100,320 cells were employed for a given flow domain in the millimeter-scale rectangular channel with the thickness of $2 \mathrm{~mm}$ (see Fig. 1). The

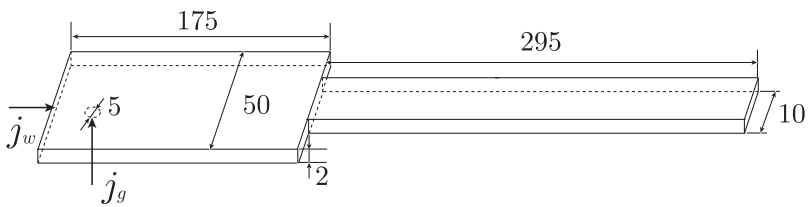

Fig. 1: Physical setup of the horizontally placed rectangular channel with an abrupt contraction (units in $\mathrm{mm}$ ). 


\begin{tabular}{lll} 
& $\theta_{c}=30^{\circ}$ & $\theta_{c}=147^{\circ}$ \\
\hline$j_{g}=20 \mathrm{~cm} / \mathrm{s}, j_{w}=28 \mathrm{~cm} / \mathrm{s}$ & case (I) & case (III) \\
$j_{g}=80 \mathrm{~cm} / \mathrm{s}, j_{w}=10 \mathrm{~cm} / \mathrm{s}$ & case (II) & case (IV) \\
\hline
\end{tabular}

Table I: Computational parameters.

superficial velocities of water (denoted by $j_{w}$ ) and air (denoted by $j_{g}$ ) were given at the left side surface having $50 \mathrm{~W} \times 2 \mathrm{H} \mathrm{mm}$ ( $W$ and $H$ indicate the width and height of the channel, respectively) and at the circular hole nozzle having $\phi 5 \mathrm{~mm}$ diameter on the bottom surface at $22.5 \mathrm{~mm}$ from the left side wall, respectively (see Fig. 1). Similar to the associated study [1], the mixed two-phase flow injected from the inlets passes through the section of the abrupt contraction and ends up outflowing from the computational domain. At the right side surface the total pressure was enforced to converge to the gauge pressure. In the present computation, two sets of superficial velocities were selected against two values of the equilibrium contact angle $\theta_{c}$ as shown in Table I. In the present situation, the flow behaves as a laminar. Similar to the associated paper [1], the wettability is called poor for $\theta_{c} \geq 90^{\circ}$ whereas called good for $\theta_{c}<90^{\circ}$.

\section{Results}

Figure 2 shows a comparison of flow patterns downstream of a circular hole nozzle, between cases (I) and (II), where the first bubble passes through the contraction. Although the superficial velocity $j_{g}$ affects the flow patterns upstream of the contraction, the bubble behavior downstream of the contraction seems to be similar between cases (I) and (II). For reference, preliminary experimental result [2] using a longer rectangular duct with the same contraction ratio is

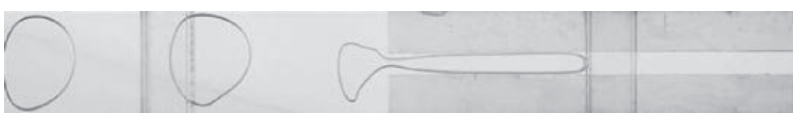

Fig. 3: Preliminary experimental result for $j_{g}=5 \mathrm{~cm} / \mathrm{s}$ and $j_{w}=10$ $\mathrm{cm} / \mathrm{s}$ using a longer rectangular duct with the same contraction ratio [2].

displayed in Fig. 3, although unfortunately the values of $j_{g}$ and $j_{w}$ are not the same $\left(j_{g}=5 \mathrm{~cm} / \mathrm{s}\right.$ and $\left.j_{w}=10 \mathrm{~cm} / \mathrm{s}\right)$. In spite of the difference in the flow rate condition, the bubble behavior at the abrupt contraction seems to be very similar between Fig. 2 (a) and Fig. 3, and this computation would be valid.

Figure 4 shows a comparison of flow patterns downstream of a circular hole nozzle, between cases (III) and (IV), under the hydrophobic condition. As seen in Fig. 4 (a), the flow pattern is very similar to the hydrophilic case of Fig. 2 (a) under the low airflow rate condition. In the high airflow rate of Fig. 4 (b), the injected air and water phases are completely mixed upstream of the contraction, and therefore the flow pattern seems to be mixed as well downstream of the contraction. This mixed flow is caused by the fact that the injected bubbles attach on the poorly wetted wall and break off into many parts.

\section{Concluding remarks}

This paper implemented the FLUENT computation and demonstrated two-phase flow patterns through a horizontally placed hydrophilic or hydrophobic rectangular channel with an abrupt contraction. The rectangular channel used in this study has a thickness narrower than the Laplace constant of this target system. In particular, the computed bubble behavior around the abrupt contraction seemed to be a similar nature against the preliminary
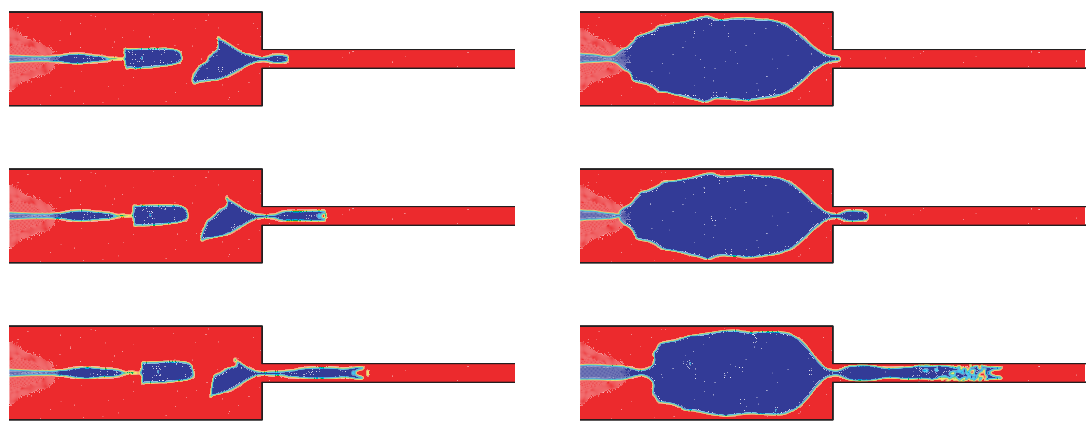

(a) case (I): $j_{g}=20 \mathrm{~cm} / \mathrm{s}, j_{w}=28 \mathrm{~cm} / \mathrm{s}$

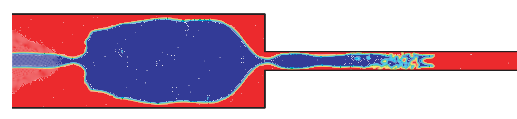

(b) case (II): $j_{g}=80 \mathrm{~cm} / \mathrm{s}, j_{w}=10 \mathrm{~cm} / \mathrm{s}$

Fig. 2: Selected snapshots of computed flow patterns for cases (I) and (II): Hydrophilic case. Blue is air phase and red is water phase. 

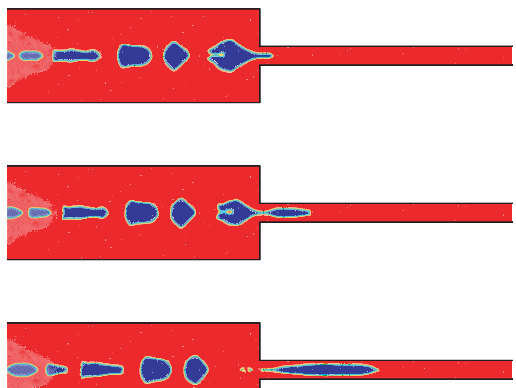

(a) case (III): $j_{g}=20 \mathrm{~cm} / \mathrm{s}, j_{w}=28 \mathrm{~cm} / \mathrm{s}$
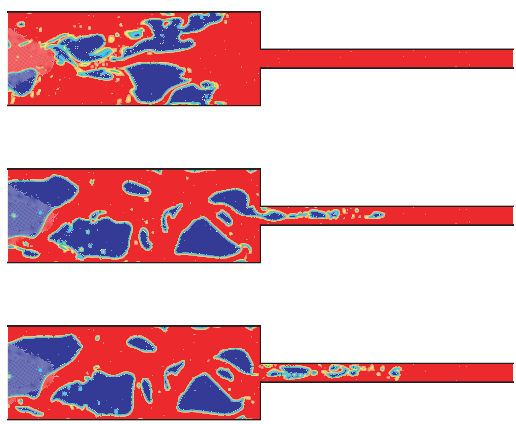

(b) case (IV): $j_{g}=80 \mathrm{~cm} / \mathrm{s}, j_{w}=10 \mathrm{~cm} / \mathrm{s}$

Fig. 4: Selected snapshots of computed flow patterns for cases (III) and (IV): Hydrophobic case. Blue is air phase and red is water phase.

experimental photograph (but not the same condition). In the computational results, the injected bubbles attach on the poorly wetted wall and break off into many parts, although they behave like slug bubbles for the wetted wall.

In near future, we intend to carry out the corresponding experiment to exactly validate the present computation and investigate more detailed phenomenon.

\section{References}

[1] Y. Ueda, T. Nakajima, T. Ishii, R. Tsujino and M. Iguchi, Submitted to High Temperature Materials and Processes.

[2] H. Fukazawa, T. Kumagai and M. Iguchi, Private Communication.

Received: May 25, 2012. Accepted: July 10, 2012. 
Brought to you by | De Gruyter / TCS

Authenticated || 173.9.48.25 